\title{
ON EXPONENTIAL SUMS OVER PRIME NUMBERS
}

\author{
A. SARKÖZY and C. L. STEWART
}

(Received 3 January 1987; revised 18 November 1987)

Communicated by J. H. Loxton

\begin{abstract}
In this article we establish an estimate for a sum over primes that is the analogue of an estimate for a sum over consecutive integers which has proved to be very useful in applications of exponential sums to problems in number theory.
\end{abstract}

1980 Mathematics subject classification (Amer. Math. Soc.) (1985 Revision): 11 L 40.

\section{Notation}

Let $c_{0}, c_{1}, \ldots$ denote effectively computable positive absolute constants. For any real number $A$, we write $\min (A, 1 / 0)=A$. For any real number $x$ let $[x]$ denote the greatest integer less than or equal to $x$, let $\{x\}=x-[x]$ denote the fractional part of $x$ and let $\|x\|=\min (\{x\}, 1-\{x\})$ denote the distance from $x$ to the nearest integer. We write $e^{2 \pi i x}=e(x)$. Further, for any positive integer $n$ let $\phi(n)$ denote the number of positive integers less than or equal to $n$ and coprime with $n$.

The research of the second author was supported in part by Grant A3528 from the Natural Sciences and Engineering Research Council of Canada.

(C) 1989 Australian Mathematical Society 0263-6115/89\$A2.00+0.00 


\section{Introduction}

In number theoretical applications of exponential sums we often use estimates for sums of the form

$$
\sum_{n \leq N} \min \left(y,\|n \alpha\|^{-1}\right)
$$

where $y$ and $\alpha$ are real numbers and $N$ is a positive integer (see, for example, [5, page 24]). The purpose of this paper is to derive similar estimates for sums of the form

$$
\sum_{p \leq N} \min \left(y,\|p \alpha\|^{-1}\right),
$$

where the summation is taken over primes instead of consecutive integers. We expect our estimates will be widely applicable. In fact, a problem in additive number theory (see [3]) first led us to the study of sums of the form (2). By using the result below we are able to simplify the proof of the main theorem of [3].

THEOREM. Let $\varepsilon$ be a positive real number. There exists an effectively computable positive absolute constant $c_{1}$ and a positive real number $N_{0}$ which is effectively computable in terms of $\varepsilon$ such that if $N$ is a positive integer with $N>N_{0}$ and $y$ is a real number with

$$
3 \leq y \leq N^{\frac{1}{4}-\varepsilon},
$$

then

$$
\sum_{p \leq N} \min \left(y,\|p \alpha\|^{-1}\right)<c_{1} \frac{N \log y \log \log y}{\log N},
$$

for all real numbers $\alpha$ with $1 / N \leq \alpha \leq 1-1 / N$.

This paper is devoted to a proof of the above theorem. We shall use some ideas from [3]. In particular the treatment of the "major arcs" will be nearly the same as in [3].

\section{Preliminary lemmas}

LEMMA 1. There exists an effectively computable positive real number $c_{2}$ such that

for $n \geq 3$.

$$
\phi(n)>c_{2} \frac{n}{\log \log n}
$$

Proof. See [2, page 24]. 
LeMmA 2. There exists an effectively computable positive real number $c_{3}$ such that for any integers $a$ and $b$ with $b \geq 2$,

$$
\sum_{\substack{1 \leq n \leq b \\(n+a, \bar{b})=1}} \frac{1}{n}<c_{3} \frac{\phi(b)}{b} \log b .
$$

Proof. This is [3, Lemma 5].

LEMMA 3. Let $h, a$ and $q$ be integers with $a>0, q>1$ and $(a, q)=1$. Let $\rho(n)$ be a real valued function defined for those integers $n$ with $h \leq n<h+q$ and $(n, q)=1$. Put

$$
\lambda=\max _{\substack{h \leq n<h+q \\(n, q)=1}} \rho(n)-\min _{\substack{h \leq n<h+q \\(n, q)=1}} \rho(n)
$$

and

$$
\psi(n)=\frac{1}{q}(a n+\rho(n)) .
$$

There is an effectively computable positive absolute constant $c_{4}$ such that if $\lambda \leq 1$ and if $E$ is a real number satisfying $2 \leq E \leq q$ then

$$
\sum_{\substack{h \leq n<h+q \\(n, q)=1}} \min \left(E, \frac{1}{\|\psi(n)\|}\right)<c_{4} \phi(q) \log E .
$$

Proof. This is [3, Lemma 6].

LeMMA 4. Let $\delta$ be a real number satisfying $0<\delta \leq 1 / 2$. Then there exists a periodic function $\psi(x, \delta)$, with period 1 , such that

(i) $\psi(x, \delta) \geq 1$ in the integral $-\delta \leq x \leq \delta$,

(ii) $\psi(x, \delta) \geq 0$ for all $x$,

(iii) $\psi(x, \delta)$ has a Fourier series of the form

$$
\psi(x, \delta)=a_{0}+\sum_{0<j \leq(1 / 2 \delta)-1} a_{j} \cos 2 \pi j x
$$

where $\left|a_{0}\right| \leq \pi^{2} \delta$ and $\left|a_{j}\right|<2 \pi^{2} \delta$ for $0<j \leq(1 / 2 \delta)-1$.

Proof. Put $N=[1 / 2 \delta]$ and

$$
\psi(x, \delta)=\frac{\pi^{2}}{4 N^{2}}\left|\sum_{k=1}^{N} e(k x)\right|^{2} .
$$


Then (ii) holds trivially. Certainly $|1-e(\alpha)|=2|\sin \pi \alpha|$ and $|\sin \alpha| \leq|\alpha|$ for all $\alpha$, while $|\sin \alpha| \geq 2|\alpha| / \pi$ for $|\alpha| \leq \pi / 2$. Therefore for $|x| \leq \delta \leq 1 /(2 N)$ we have

$$
\psi(x, \delta)=\frac{\pi^{2}}{4 N^{2}}\left|\frac{1-e(N x)}{1-e(x)}\right|^{2}=\frac{\pi^{2}}{4 N^{2}} \frac{|\sin \pi N x|^{2}}{|\sin \pi x|^{2}} \geq \frac{\pi^{2}}{4 N^{2}} \frac{\left(\frac{2}{\pi} \cdot \pi N x\right)^{2}}{(\pi x)^{2}}=1,
$$

and so (i) also holds.

Finally, we have

$$
\begin{aligned}
\psi(x, \delta) & =\frac{\pi^{2}}{4 N^{2}}\left|\sum_{k=1}^{N} e(k x)\right|^{2}=\frac{\pi^{2}}{4 N^{2}} \sum_{k=1}^{N} e(k x) \sum_{l=1}^{N} e(-l x) \\
& =\frac{\pi^{2}}{4 N^{2}}\left(N+\sum_{j=1}^{N-1}(N-j)(e(j \alpha)+e(-j \alpha))\right) \\
& =\frac{\pi^{2}}{4 N}+\sum_{j=1}^{N-1} \frac{\pi^{2}(N-j)}{2 N^{2}} \cos j \alpha=a_{0}+\sum_{0<j \leq N-1} a_{j} \cos j \alpha \\
& =a_{0}+\sum_{0<j \leq(1 / 2 \delta)-1} a_{j} \cos j \alpha
\end{aligned}
$$

where

$$
a_{0}=\frac{\pi^{2}}{4 N}=\frac{\pi^{2}}{4[1 / 2 \delta]} \leq \frac{\pi^{2}}{2(1 / 2 \delta)}=\pi^{2} \delta
$$

and

$$
a_{j}=\frac{\pi^{2}(N-j)}{2 N^{2}}<\frac{\pi^{2} N}{2 N^{2}}=\frac{\pi^{2}}{2 N}=2 a_{0} \leq 2 \pi^{2} \delta \quad \text { for } 0<j \leq(1 / 2 \delta)-1,
$$

which completes the proof of Lemma 4.

We shall also require the Brun-Titchmarsh theorem and a refinement, due to Vaughan, of Vinogradov's fundamental lemma.

Let $x$ be a positive real number and let $l$ and $k$ be positive integers. As usual we denote the number of primes less than or equal to $x$ by $\pi(x)$, and the number of primes less than or equal to $x$ and congruent to $l$ modulo $k$ by $\pi(x, k, l)$.

LEMMA 5 (Brun-Titchmarsh theorem). Let $x$ and $y$ be positive real numbers and let $k$ and $l$ be relatively prime positive integers with $y>k$. Then

$$
\pi(x+y, k, l)-\pi(x, k, l)<\frac{2 y}{\phi(k) \log (y / k)}
$$

Proof. See [1, Theorem 2]. 
LEMMA 6. If $\alpha$ is a real number and $a, q, H$ and $N$ are positive integers with $(a, q)=1, q \leq N, H<N$ and $|\alpha-a / q| \leq q^{-2}$ then

$$
\begin{aligned}
\sum_{h=1}^{H}\left|\sum_{p \leq N} e(h \rho \alpha)\right|<c_{5}(\log N)^{6}\left(H N q^{-1 / 2}+H N^{3 / 4}+(H N q)^{1 / 2}\right. \\
\left.+H^{3 / 5} N^{4 / 5} \exp (2 \log N / \log \log N)\right),
\end{aligned}
$$

where $c_{5}$ is an effectively computable positive absolute constant.

Proof. This follows from [2, Satz 5.2] and [4, Theorem 1] by partial summation.

\section{Further preliminaries}

Put $P=y^{2}(\log N)^{14}$ and $Q=N / P$.

Let $T_{1}$ denote the set of those $\alpha$ in the interval $(1 / N, 1-1 / N)$ for which there exist positive integers $a$ and $b$ with $(a, b)=1$, such that

$$
\left|\alpha-\frac{a}{b}\right|<\frac{1}{b^{2}}
$$

and

$$
P \leq b \leq Q=N / P .
$$

Put $T^{\prime}=(1 / N, 1-1 / N)-T_{1}$, so that $T^{\prime}$ consists of the real numbers $\alpha$ in $(1 / N, 1-1 / N)$ which are not in $T_{1}$. Suppose that $\alpha \in T^{\prime}$. Then by Dirichlet's theorem there exist integers $a$ and $b$ with

$$
\left|\alpha-\frac{a}{b}\right| \leq \frac{1}{b Q},
$$

$0 \leq a, 0<b<Q$ and $(a, b)=1$. Plainly

$$
\left|\alpha-\frac{a}{b}\right|<\frac{1}{b^{2}}
$$

and thus,

$$
0<b<P \text {. }
$$

To each $\alpha$ in $T^{\prime}$ we shall associate a pair of coprime integers $a$ and $b$ with $a \geq 0$ and $b>0$ satisfying (6) and (8) and we shall put $\beta=\alpha-\frac{a}{b}$. Let us define subsets $T_{2}, T_{3}$ and $T_{4}$ of $T^{\prime}$ in the following way:

$$
\begin{aligned}
& T_{2}=\left\{\alpha \in T^{\prime}: 1 \leq b \leq y,|\beta| \leq 1 / 2 b N\right\}, \\
& T_{3}=\left\{\alpha \in T^{\prime}: 1 \leq b \leq y,|\beta|>1 / 2 b N\right\}, \\
& T_{4}=\left\{\alpha \in T^{\prime}: y<b\right\} .
\end{aligned}
$$


Further put

$$
S(\alpha)=\sum_{p \leq N} \min \left(y,\|p \alpha\|^{-1}\right) .
$$

Since $(1 / N, 1-1 / N)=T_{1} \cup T_{2} \cup T_{3} \cup T_{4}$ it suffices to show that

$$
\max _{\alpha \in T_{i}} S(\alpha)<c_{6} \frac{N \log y \cdot \log \log y}{\log N}
$$

for $i=1,2,3,4$ when $N>N_{0}$. For $i=1$ ("minor arcs"), (9) will be established in Section 5, while cases $i=2,3,4$ ("major arcs") will be dealt with in Section 6.

\section{Minor arcs}

Assume that $\alpha \in T_{1}$ and let $N_{0}, N_{1}, N_{2}, \ldots$ denote real numbers which are effectively computable in terms of $\varepsilon$.

For $\beta>0$, put

$$
Z(N, \alpha, \beta)=\sum_{\substack{p \leq N \\\|p \alpha\|<\beta}} 1 .
$$

Then by the prime number theorem, for $N>N_{1}$,

$$
\begin{aligned}
S(\alpha) & =\sum_{p \leq N} \min \left(y,\|p \alpha\|^{-1}\right) \\
& =\sum_{\substack{p \leq N \\
\|p \alpha\|<1 / y}} \min \left(y,\|p \alpha\|^{-1}\right)+\sum_{j=2}^{[y / 2]+1} \sum_{\substack{p \leq N \\
\frac{j-1}{y} \leq\|p \alpha\|<\frac{j}{y}}} \min \left(y,\|p \alpha\|^{-1}\right) \\
& \leq \sum_{\substack{p \leq N \\
\|p \alpha\|<1 / y}} y+\sum_{j=2}^{[y / 2]+1} \sum_{\substack{p \leq N \\
\frac{i-1}{y} \leq\|p \alpha\|<\frac{j}{y}}} \min \left(y,\left(\frac{j-1}{y}\right)^{-1}\right) \\
& =y Z(N, \alpha, 1 / y)+\sum_{j=2}^{[y / 2]+1} \frac{y}{j-1}(Z(N, \alpha, j / y)-Z(N, \alpha,(j-1) / y)) \\
& =y \sum_{j=2}^{[y / 2]} Z(n, \alpha, j / y)\left(\frac{1}{j-1}-\frac{1}{j}\right)+\frac{y}{[y / 2]} Z(N, \alpha,([y / 2]+1) / y) \\
& \leq y \sum_{j=2}^{[y / 2]} \frac{Z(n, \alpha, j / y)}{(j-1) j}+4 \sum_{p \leq N} 1 \\
& <y \sum_{j=2}^{[y / 2]} \frac{Z(N, \alpha, j / y)}{(j-1) j}+5 \frac{N}{\log N} .
\end{aligned}
$$


By Lemma 4 (with $j / y$ in place of $\delta$ ), for $N>N_{2}$ and $1 \leq j \leq y / 2$ we have

$$
\begin{aligned}
Z(N, \alpha, j / y) & =\sum_{\substack{p \leq N \\
\|p \alpha\|<j / y}} 1 \leq \sum_{p \leq N} \psi(p \alpha, j / y) \\
& =\sum_{p \leq N}\left(a_{0}+\sum_{0<k \leq(y / 2 j)-1} a_{k} \cos 2 \pi k(p \alpha)\right) \\
& =a_{0} \pi(N)+\sum_{0<k \leq(y / 2 j)-1} a_{k} \operatorname{Re} \sum_{p \leq N} e(k p \alpha) \\
& \leq\left|a_{0}\right| \pi(N)+\sum_{0<k \leq(y / 2 j)-1}\left|a_{k}\right|\left|\sum_{p \leq N} e(k p \alpha)\right| \\
& \leq 2 \pi^{2} \frac{j}{y}\left(\frac{N}{\log N}+\sum_{0<k \leq(y / 2 j)-1}\left|\sum_{p \leq N} e(k p \alpha)\right|\right) .
\end{aligned}
$$

Thus, by Lemma 6,

$$
\begin{gathered}
Z(N, \alpha, j / y) \\
<20 \frac{j}{y}\left(\frac{N}{\log N}+c_{5}(\log N)^{6}\left(\frac{y}{2 j} N P^{-1 / 2}+\frac{y}{2 j} N^{3 / 4}\left(\frac{y}{2 j} \frac{N^{2}}{P}\right)^{1 / 2}\right.\right. \\
\left.\left.+\left(\frac{y}{2 j}\right)^{3 / 5} N^{4 / 5} \exp (2 \log N / \log \log N)\right)\right) \\
<20 \frac{j}{y}\left(\frac{N}{\log N}+c_{5}(\log N)^{6}\left(2 y N P^{-1 / 2}+y N^{3 / 4}+y^{3 / 5} N^{4 / 5}\right.\right. \\
\exp (2 \log N / \log \log N))) .
\end{gathered}
$$

Since $P=y^{2}(\log N)^{14}$ it follows from (3) and (11) that for $N>N_{3}$ and $1 \leq j \leq y / 2$

$$
Z(N, \alpha, j / y)<20 \frac{j}{y} \frac{N}{\log N}+c_{6} \frac{j}{y} \frac{N}{\log N}<c_{7} \frac{j}{y} \frac{N}{\log N} .
$$

Thus from (10),

$$
\begin{aligned}
S(\alpha) & <y \sum_{j=2}^{[y / 2]} \frac{1}{(j-1) j} \cdot c_{7} \frac{j}{y} \frac{N}{\log N}+5 \frac{N}{\log N} \\
& <5 \frac{N}{\log N}+c_{7} \frac{N}{\log N} \sum_{j=2}^{[y / 2]} \frac{1}{j-1}<c_{8} \frac{N \log y}{\log N} \quad\left(\text { for } \alpha \in T_{1}\right) .
\end{aligned}
$$




\section{Major arcs}

Put

$$
S(\alpha, b)=\sum_{\substack{p \leq N \\(p, b)=1}} \min \left(y,\|p \alpha\|^{-1}\right)
$$

In view of (3) for $N>N_{5}$ we have for any real number $\alpha$ and positive integer $b \leq N$, that

$$
\begin{aligned}
S(\alpha) & =\sum_{p \leq N} \min \left(y,\|p \alpha\|^{-1}\right) \\
& \leq \sum_{p \mid b} y+\sum_{\substack{p \leq N \\
(p, b)=1}} \min \left(y,\|p \alpha\|^{-1}\right) \\
& =y \sum_{p \mid b} 1+S(\alpha, b)<c_{9} y \log b+S(\alpha, b) \\
& \leq c_{9} y \log N+S(\alpha, b)<\frac{N}{\log N}+S(\alpha, b) .
\end{aligned}
$$

Assume first that $\alpha \in T_{2}$. Notice that we may assume that $b>1$, since if $b=1$ then $|\beta| \leq 1 / 2 N$ and consequently $\alpha$ is not in $(1 / N, 1-1 / N)$. Further since $b \neq 1$ we may assume that $a \neq 0$.

For $(p, b)=1$ we have

$$
\begin{aligned}
\|p \alpha\| & =\left\|p\left(\frac{a}{b}+\beta\right)\right\| \geq\left\|\frac{a p}{b}\right\|-p|\beta| \geq\left\|\frac{a p}{b}\right\|-N \frac{1}{2 b N} \\
& =\left\|\frac{a p}{b}\right\|-\frac{1}{2 b} \geq \frac{1}{2}\left\|\frac{a p}{b}\right\|
\end{aligned}
$$

since $b>1$ and $(a p, b)=1$. Thus

$$
\begin{aligned}
S(\alpha, b) & \leq \sum_{\substack{p \leq N \\
(p, b)=1}} \min \left(y, 2\|a p / b\|^{-1}\right) \\
& =\sum_{\substack{0 \leq h<b \\
(h, b)=1}} \sum_{\substack{p \leq N \\
a p=h(\bmod b)}} 2\|h / b\|^{-1} \\
& \leq 2\left(\max _{\substack{0<l<b \\
(l, b)=1}} \pi(N, b, l)\right) \sum_{\substack{0 \leq h<b \\
(h, b)=1}}\|h / b\|^{-1} .
\end{aligned}
$$

By Lemma 5, (3) and $b \leq y$, we have

$$
S(\alpha, b) \leq \frac{4 N}{\phi(b) \log (N / b)} 2 \sum_{\substack{1 \leq h \leq b / 2 \\(h, b)=1}} \frac{b}{h} \leq \frac{11 N}{\phi(b) \log N} \sum_{\substack{1 \leq h \leq b / 2 \\(h, b)=1}} b / h
$$


and so, by Lemma 2 ,

$$
S(\alpha, b) \leq c_{10} \frac{N \log b}{\log N} \leq c_{10} \frac{N \log y}{\log N} \quad\left(\text { for } \alpha \in T_{2}\right)
$$

as required.

We shall assume next that $\alpha \in T_{3}$, whence

$$
\frac{1}{2 b N}<|\beta| \leq \frac{1}{b Q}
$$

Put $L=1 / 2 b|\beta|$. It follows from (15) that

$$
\frac{Q}{2} \leq L<N
$$

We have

$$
\begin{aligned}
S(\alpha, b) & =\sum_{\substack{p \leq N \\
(p, b)=1}} \min \left(y,\|p \alpha\|^{-1}\right) \\
& \leq \sum_{j=1}^{[N / L]+1} \sum_{\substack{(j-1) L<p \leq j L \\
(p, b)=1}} \min \left(y,\|p \alpha\|^{-1}\right) \\
& =\sum_{j=1}^{[N / L]+1} \sum_{k=1}^{2 y} \sum_{\substack{(j-1) L<p \leq j L \\
(p, b)=1 \\
\frac{k-1}{2 y} \leq\{p \alpha\}<\frac{k}{2 y}}} \min \left(y,\|p \alpha\|^{-1}\right) .
\end{aligned}
$$

Since $(k-1) /(2 y) \leq\{p \alpha\}<k /(2 y)$ implies that

$$
\frac{1}{\|p \alpha\|} \leq \frac{1}{\left\|\frac{k-1}{2 y}\right\|}+\frac{1}{\left\|\frac{k}{2 y}\right\|}
$$

where, as before, we write $a \leq 1 / 0+b$ and $1 / 0 \leq 1 / 0+a$ for all real numbers $a$ and $b$, we have

$$
\begin{aligned}
S(\alpha, b) \leq \sum_{j=1}^{[N / L]+1} \sum_{k=1}^{2 y}(\min (y, & \left.\left\|\frac{k-1}{2 y}\right\|^{-1}\right) \\
& \left.+\min \left(y,\left\|\frac{k}{2 y}\right\|^{-1}\right)\right) \sum_{\substack{(j-1) L<p \leq j L \\
\frac{k-1}{2 y} \leq\{p \alpha\}<\frac{k}{2 y} \\
(p, b)=1}} 1 .
\end{aligned}
$$


If $p$ and $p_{0}$ are primes with $(j-1) L<p \leq j L,(k-1) /(2 y) \leq\{p \alpha\}<(k / 2 y)$ and $(j-1) L<p_{0} \leq j L,(k-1) /(2 y) \leq\left\{p_{0} \alpha\right\}<k /(2 y)$ then

$$
\begin{aligned}
\frac{1}{2 y} & >\left\|\left(p-p_{0}\right) \alpha\right\|=\left\|\left(p-p_{0}\right)\left(\frac{a}{b}+\beta\right)\right\| \geq\left\|\left(p-p_{0}\right) \frac{a}{b}\right\|-\left|p-p_{0} \| \beta\right| \\
& >\left\|\left(p-p_{0}\right) \frac{a}{b}\right\|-L|\beta|=\left\|\left(p-p_{0}\right) \frac{a}{b}\right\|-\frac{1}{2 b} .
\end{aligned}
$$

Thus $\left\|\left(p-p_{0}\right) a / b\right\|<1 / 2 y+1 /(2 b) \leq 1 / b$, whence $p \equiv p_{0}(\bmod b)$. Therefore

$$
\frac{1}{2 y}>\left\|p \alpha-p_{0} \alpha\right\|=\left\|\left(p-p_{0}\right) \frac{a}{b}+\left(p-p_{0}\right) \beta\right\|=\left\|\left(p-p_{0}\right) \beta\right\| .
$$

Since $\left|\left(p-p_{0}\right) \beta\right|<L|\beta|=1 /(2 b) \leq 1 / 2$, it follows from (18) that $1 /(2 y)>$ $\left|p-p_{0}\right||\beta|$, and hence

$$
\left|p-p_{0}\right|<\frac{1}{2|\beta| y} \text {. }
$$

Thus, either there are no primes $p$ with $(j-1) L<p \leq j L,(p, b)=1$ and $(k-1) /(2 y) \leq\{p \alpha\}<k /(2 y)$, or for some $p_{0}$ we have

$$
\begin{aligned}
& \sum_{\substack{(j-1) L<p \leq j L \\
(p, b)=1 \\
\frac{k-1}{2 y} \leq\{p \alpha\}<\frac{k}{2 y}}} 1 \leq \sum_{\substack{p_{0}-\frac{1}{2|\beta| y}<p<p_{0}+\frac{1}{2|\beta| y} \\
p \equiv p_{0}(\bmod b)}} 1 \\
& \leq \pi\left(p_{0}+\frac{1}{2|\beta| y}, b, p_{0}\right)-\pi\left(p_{0}-\frac{1}{2|\beta| y}, b, p_{0}\right) .
\end{aligned}
$$

By (15), $1 /(|\beta| y) \geq b Q / y$. Thus, for $N>N_{6}$, the right-hand side of inequality (19) is, by (3) and Lemma 5 , at most

$$
\frac{\frac{2}{|\beta| y}}{\phi(b) \log \frac{1}{|\beta| y b}} \leq \frac{4 b L}{y \phi(b) \log \frac{Q}{y}} \leq c_{11} \frac{b L}{y \phi(b) \log N} .
$$

In view of (16) it now follows from (17), that

$$
\begin{aligned}
S(\alpha, b) & \leq \sum_{j=1}^{[N / L]+1} \sum_{k=1}^{2 y}\left(\min \left(y,\left\|\frac{k-1}{2 y}\right\|^{-1}\right)\right. \\
& \left.\quad+\min \left(y,\left\|\frac{k}{2 y}\right\|^{-1}\right)\right) c_{11} \frac{b L}{y \phi(b) \log N} \\
& \leq\left(\left[\frac{N}{L}\right]+1\right) c_{12} \frac{b L}{y \phi(b) \log N} \sum_{k=0}^{y} \min \left(y,\left\|\frac{k}{2 y}\right\|^{-1}\right) \\
& \leq c_{13} \frac{N b}{y \phi(b) \log N}\left(y+\sum_{k=1}^{y} \frac{2 y}{k}\right) \leq c_{14} \frac{N b \log y}{\phi(b) \log N} .
\end{aligned}
$$


Thus, by Lemma $1, S(\alpha, b) \leq c_{15} N \log y \log \log b / \log N$. Since $b \leq y$ we have

$$
S(\alpha, b) \leq c_{15} \frac{N \log y \log \log y}{\log N} \quad\left(\text { for } \alpha \in T_{3}\right)
$$

provided that $N>N_{7}$.

Finally we assume that $\alpha \in T_{4}$. Put $M=\min (N, 1 /(|\beta| y))$. Then

$$
\begin{aligned}
S(\alpha, b) & =\sum_{\substack{p \leq N \\
(p, b)=1}} \min \left(y,\|p \alpha\|^{-1}\right) \\
& \leq \sum_{j=1}^{[N / M]+1} \sum_{\substack{(j-1) M<p \leq j M \\
(p, b)=1}} \min \left(y,\|p \alpha\|^{-1}\right) .
\end{aligned}
$$

Now if $\|p \alpha\|^{-1}<y$ with $(j-1) M<p \leq j M$ and $n$ is defined by $p \equiv n$ $(\bmod b)$ with $j M-b<n \leq j M$ then

$$
\|p \alpha\|=\left\|p\left(\frac{a}{b}+\beta\right)\right\|=\left\|\frac{a n}{b}+n \beta+(p-n) \beta\right\| \geq\left\|\frac{1}{b}(a n+n b \beta)\right\|-|p-n \| \beta| .
$$

Since $b>y$ it follows from (6) that $1 /(|\beta| y)>Q$, and hence, for $N>N_{8}$, that $M>Q$. Consequently $b<M$ and so $|p-n|<M$ and $|p-n||\beta|<$ $M|\beta| \leq 1 / y<\|p \alpha\|$. Thus $2\|p \alpha\| \geq\|(1 / b)(a n+n b \beta)\|$, and hence

$$
\begin{aligned}
\min \left(y,\|p \alpha\|^{-1}\right) & \leq \min \left(y, 2\left\|\frac{1}{b}(a n+n b \beta)\right\|^{-1}\right) \\
& \leq 2 \min \left(y,\left\|\frac{1}{b}(a n+n b \beta)\right\|^{-1}\right) .
\end{aligned}
$$

Therefore, by (21),

$$
S(\alpha, b) \leq \sum_{j=1}^{[N / M]+1} \sum_{\substack{j M-b<n \leq j M \\(n, b)=1}} 2 \min \left(y,\left\|\frac{1}{b}(a n+n b \beta)\right\|^{-1}\right) \sum_{\substack{(j-1) M<p \leq j M \\ p \equiv n(\bmod b)}} 1 .
$$

For $N>N_{9}$ we have, by (3) and (8), that

$$
\frac{M}{b}>\frac{Q}{b}>\frac{N}{P^{2}}=\frac{N}{y^{4}(\log N)^{28}}>N^{2 \varepsilon},
$$

whence, from Lemma 5 ,

$$
\sum_{\substack{(j-1) M<p \leq j M \\ p \equiv n(\bmod b)}} 1<\frac{2 M}{\phi(b) \log \frac{M}{b}} \leq \frac{M}{\varepsilon \phi(b) \log N} .
$$


Combining (22) and (24), we obtain

$$
S(\alpha, b) \leq \frac{M}{\varepsilon \phi(b) \log N} \sum_{j=1}^{[N / M]+1} \sum_{\substack{j M-b<n \leq j M \\(n, b)=1}} \min \left(y,\left\|\frac{1}{b}(a n+n b \beta)\right\|^{-1}\right) .
$$

We may estimate the inner sum above by means of Lemma 3 with $h=$ $j M-b+1, q=b$ and $\rho(n)=n b \beta$. Then by (6) and (23),

$$
\lambda=\max _{\substack{j M-b<n \leq j M \\(n, b)=1}} n b \beta-\min _{\substack{j M-b<n \leq j M \\(n, b)=1}} n b \beta \leq b^{2}|\beta|<\frac{b}{Q}<N^{-2 \varepsilon}<1
$$

for $N>N_{10}$. Thus

$$
S(\alpha, b) \leq \frac{M}{\varepsilon \phi(b) \log N}\left(\left[\frac{N}{M}\right]+1\right) c_{4} \phi(b) \log y,
$$

and, since $M \leq N$,

$$
S(\alpha, b) \leq c_{16} \frac{N \log y}{\varepsilon \log N} \quad\left(\text { for } \alpha \in T_{4}\right) .
$$

If $y<N^{1 / 10}(\log N)^{-7}$ then we may replace $2 \varepsilon$ in (23) by $1 / 2$ and consequently $\varepsilon$ in $(25)$ by 1 . On the other hand if $y \geq N^{1 / 10}(\log N)^{-7}$ then certainly $1 / \varepsilon<\log \log y$ for $N>N_{11}$. Thus in either case, we obtain from (25) that

$$
\left.S(\alpha, b) \leq c_{17} \frac{N \log y \log \log y}{\log N} \text { (for } N>N_{11}, \alpha \in T_{4}\right) \text {. }
$$

Thus (9) follows from (12), (13), (14), (20) and (26), and this completes the proof of the theorem.

\section{Addendum}

We would like to thank the referee for his valuable suggestions and remarks. In particular, the referee drew our attention to reference [4] which allowed us to improve our original exponent of $\frac{1}{5}$ in (3) to $\frac{1}{4}$.

Further, the referee remarked that our estimate for $S(\alpha)$ is essentially best possible for a special choice of $y$. In fact, by means of a slight generalization of the referee's idea, we shall show that there exist effectively computable positive constants $c_{18}$ and $c_{19}$ such that if $N>c_{18}$ then for all real numbers $y$ with $3 \leq y \leq N^{1 / 4}$ we have

$$
\max _{\frac{1}{N} \leq \alpha \leq 1-\frac{1}{N}} \sum_{p \leq N} \min \left(y,\|p \alpha\|^{-1}\right)>c_{19} \frac{N \log y \log \log y}{\log N} .
$$

Therefore our main theorem gives the correct order of magnitude for $S(\alpha)$. 
We shall now establish (26). Define the integer $x$ by

$$
\prod_{p \leq x} p \leq y^{2 / 3}<\prod_{p \leq x+1} p
$$

and put $b=\prod_{p \leq x} p$. Note that $x \geq 2$ since $y \geq 3$. We have

$$
x>c_{20} \log y
$$

and

$$
\phi(b)=b \prod_{p \leq x}\left(1-\frac{1}{p}\right)<c_{21} \frac{b}{\log x}
$$

Thus

$$
\begin{aligned}
\sum_{\substack{1 \leq a \leq b \\
(a, b)=1}} S\left(\frac{a}{b}-\frac{1}{b N}\right) & =\sum_{\substack{1 \leq a \leq b \\
(a, b)=1}} \sum_{p \leq N} \min \left(y,\left\|\frac{a p}{b}-\frac{p}{b N}\right\|^{-1}\right) \\
& =\sum_{\substack { p \leq N \\
\begin{subarray}{c}{1 \leq a \leq b \\
(a, b)=1{ p \leq N \\
\begin{subarray} { c } { 1 \leq a \leq b \\
( a , b ) = 1 } }\end{subarray}} \min \left(y,\left\|\frac{a p}{b}-\frac{p}{b N}\right\|^{-1}\right) .
\end{aligned}
$$

Since $\frac{N}{4}>N^{1 / 4}>y^{2 / 3} \geq b$ for $N>8$,

$$
\begin{aligned}
& \sum_{\substack{1 \leq a \leq b \\
(a, b)=1}} S\left(\frac{a}{b}-\frac{1}{b N}\right) \geq \sum_{\substack{N \\
\frac{N}{4} \leq p \leq N}} \sum_{\substack{1 \leq h \leq b \\
(h, b)=1}} \min \left(y,\left\|\frac{h}{b}-\frac{p}{b N}\right\|^{-1}\right) \\
& \geq \sum_{\frac{N}{4}<p \leq N} \min \left(y \cdot\left\|\frac{1}{b}-\frac{p}{b N}\right\|^{-1}\right) \\
& \geq \sum_{i=1}^{[3 y / 4 b]} \sum_{N-\frac{i b N}{y}<p \leq N-(i-1) \frac{b N}{y}} \min \left(y,\left\|\frac{1}{b}-\frac{1}{b} \frac{(N-i b N / y)}{N}\right\|^{-1}\right) \\
& \geq \sum_{i=1}^{[3 y / 4 b]} \min \left(y,\left\|\frac{i}{y}\right\|^{-1}\right)(\pi(N-(i-1) b N / y)-\pi(N-i b N / y)) .
\end{aligned}
$$

Since $\pi(x+z)-\pi(x)>c_{22} z / \log x$, for $z>x^{3 / 4}$ and $x$ sufficiently large, we find that for $N>c_{23}$,

$$
\pi\left(N-(i-1) \frac{b N}{y}\right)-\pi(N-i b N / y)>c_{24} \frac{b N}{y \log N} .
$$

Thus

$$
\sum_{\substack{1 \leq a \leq b \\(a, b)=1}} S\left(\frac{a}{b}-\frac{1}{b N}\right)>\sum_{i=1}^{[3 y / 4 b]} \frac{c_{24} b N}{i \log N}>c_{25} \frac{b N \log y}{\log N} .
$$


Therefore

$$
\max _{\substack{1 \leq a \leq b \\(a, b)=1}} S\left(\frac{a}{b}-\frac{1}{b N}\right)>c_{25} \frac{b}{\phi(b)} \frac{N \log y}{\log N}
$$

and so, by (27) and (28),

$$
\max _{\substack{1 \leq a \leq b \\(a, b)=1}} S\left(\frac{a}{b}-\frac{1}{b N}\right)>c_{26} \frac{N \log y \log \log y}{\log N},
$$

which proves (26).

Finally, the $L^{1}$ mean of $S(\alpha)$ is asymptotically $2(1+\log (y / 2)) \pi(N)$ and the referee asked whether $S(\alpha)$ has this size outside of a small set. We remark that by our proof, we have

$$
\max _{\substack{\alpha \in[0,1] \\ \alpha \notin T_{3} \\\|\alpha\|>1 / N}} S(\alpha)<c_{27} \log y \pi(N) .
$$

Further the measure of $T_{2} \cup T_{3}$ is, by (6), at most

$$
\sum_{b=1}^{[y]} \frac{\phi(b)}{b Q} \leq \frac{y}{Q}=\frac{y^{3}(\log N)^{14}}{N} .
$$

Thus (29) holds for all $\alpha$ in $[0,1]$ except for a set of measure at most $\left(2+y^{3}(\log N)^{14}\right) / N$. In fact we can be more precise if we make the minor arcs slightly smaller. For example, put $P_{1}=y^{2}(\log N)^{20}$ and $Q_{1}=N / P_{1}$. It is possible to show that $S(\alpha)$ is $2(1+\log (y / 2)) \pi(N)(1+o(1))$ for all $\alpha$ in $(1 / N, 1-1 / N)$ for which there exist coprime positive integers $a$ and $b$ with $\left|\alpha-\frac{a}{b}\right|<b^{-2}$ and $P_{1} \leq b \leq Q_{1}$. Notice that the complement of this set in $(0,1)$ has measure at most $2 / N+P_{1} / Q_{1}=\left(2+y^{4}(\log N)^{40}\right) / N$. To prove this requires a more careful analysis of $S(\alpha)$ on the minor arcs. In particular we must replace the function $\psi(x, \delta)$ with its finite Fourier series by a function that is a better approximation to the function $f$ where

$$
f(x)= \begin{cases}1 & \text { for }\|x\| \leq \delta \\ 0 & \text { for } \delta<\|x\| \leq 1 / 2\end{cases}
$$

Such a function can be found by an appropriate truncation of the Fourier series expansion of $f$.

\section{References}

[1] H. L. Montgomery and R. C. Vaughan, 'The large sieve', Mathematika 20 (1973), 119-134.

[2] K. Prachar, Primzahlverteilung (Springer-Verlag, 1957). 
[3] A. Sárközy and C. L. Stewart, 'On divisors of sums of integers, II', J. Reine Angew. Math. 365 (1986), 171-191.

[4] R. C. Vaughan, 'On the distribution of $\alpha p$ modulo 1', Mathematika 24 (1977), 135-141.

[5] I. M. Vinogradov, The method of trigonometric sums in the theory of numbers (Interscience, New York, 1954).

Mathematical Institute of the

Hungarian Academy of Sciences

Reáltanoda u. 13-16

Budapest, H-1053

Hungary
Department of Pure Mathematics

University of Waterloo Waterloo, Ontario N2L 3G1

Canada 